\section{Neglected Cellular Organelles}

Krystyna Kielan Rybicka, SUNY at Buffalo

Virtually all animal cells contain organelles called glycosomes which until now have been neglected in cell biology. This author recently published a comprehensive review article entitled "Glycosomes - the organelles of glycogen metabolism", Tissue \& Cell 28 (3) 253-265, 1996. The review, based on biochemical and microscopy data, includes a complete history of glycogen research and the current status of knowledge tence of a complex enzymatic machinery involved in glycogen synthesis and degradation, which constantly accumulates glycogen and releases glucose. Such types of cellular machineries represent cell organelles and these were already called glycosomes. There is, however, a confusion in the morphological data concerning glycosomes since early electron microscopic research incorrectly identified the protein component of glycosomes as particles of glycogen. This interpretation, accepted in textbooks and diagnostic pathology, needs an urgent revision.

The protein component in glycosomes is fixed by osmium, and stained by uranium and lead. Glycogen accumulated in glycosomes is neither fixed per sec nor stained by heavy metals. Glycogen can be visualized by special techniques such as histochemistry or negative staining. The differences in size and in the electron density of glycosomal protein stained by uranium and glycosomes rather then the amount of glycogen. Several data suggest that the large, electron dense particles may appear when phosphorylase is present (or active) in the organelle, whereas the small and poorly contrasted protein particles would imply the activity of glycogen synthase.

Furthermore, there is an intimate association between glycosomes and numerous other cellular organelles including the intermediate filaments and the membranes of endoplasmlc reticulum. The association may be related to
Biochemical studies on glycogen metabolism demonstrate the exislead (commonly called 'glycogen particles') indicates the metabolic state of

the energy release by glycosomes, as well as to the transport of glycosomes within the cell, similar to the well recognized transport of ribosomes. The understanding of the structure of glycoscmes opens a vast field for the application of modern molecular and cellular biology techniques in order to study cellular $\overrightarrow{.}$ metabolism of glycosomes and the rote of these ubiquitous organelles in the cell.

I would be very happy to receive some feedback on the ideas I outlined in my review article and to continue discussion with anyone interested in glycogen research and in the integrated research on the cell. I will be at the MSA Conference in Cleveland or may be contacted by eMail:

rybicka@acsu.buffalo.edu

\section{Readers, Please Note:}

Potential advertisers have an understandable interest in the background and interesty of readers of a publication as they consider placement of their advertising dollars. For this reason, it is important to me to have an accurate summary of the interesty of our readership.

For this reason, and if you have not already done so, I request that you complete the interest profile on the enclosed, postage paid, reader response card and return it to me.

Should you not be sure if you have previously completed the survey, kindly complete the survey. I would much rather receive two responses from you rather than none. Many thanks for your help!

\section{-. Don Grimes, Editor}

\section{Bonjour...Buenos Dias...Guten Tag Buon giorno... Konnichi wa}

\section{Goedendag}

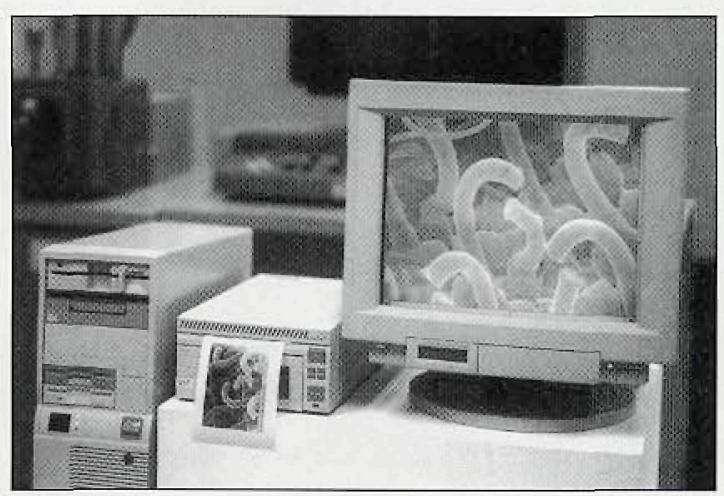

- ACTIVE BEAM CONTROL

- UP TO $4096 \times 4096$ CAPTURE (12-BIT Digitization)

- DUAL ADCS (Collect Secondary and Backscatter

- FRAME and PIXEL AVERAGING
- AUTOMATIC CONTROL CONTRAST/BRIGITNESS

- OPTIONAL. FAST X-RAY MAPPING

- OPTIONAL IMAGE ANALYSIS SOFTWARE

\section{Vital Image Technology • 800-860-IMAGE}

Vital Image Technology offers photographic-quality printers and other imaging related products such as CCD cameras, frame grabbers and scanners. VIT has the imasing solution for both network and stand alone applications.
Ampersand International, Inc.

111 Twin Creek Lane - Freedom, PA 15042 Telephone (412) $742-4420 \cdot \mathrm{Fax}$ (412) $742-4430$ E-Mail: amprsand $@$ nauticom net 


\section{Don't find rourself in a}

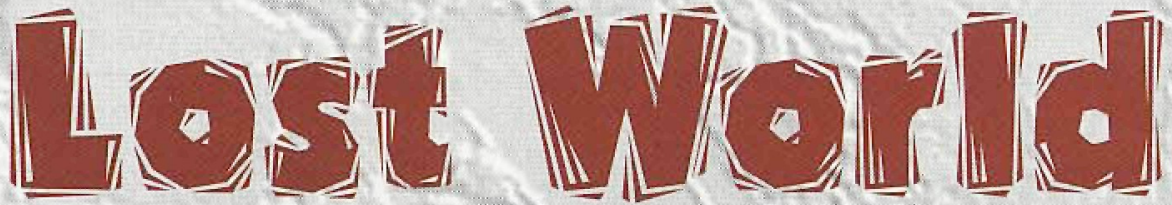

\section{with a Jurassic EDS system.}

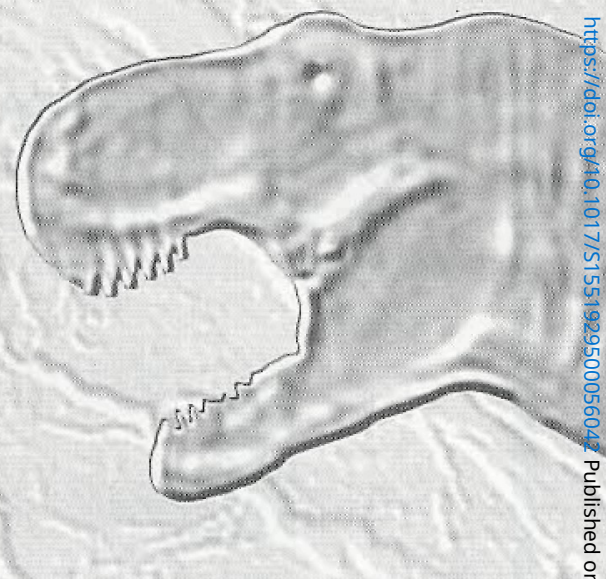

\section{Enter the 21st Century with cutting edge technology Quantum EXiralliluti and SYS4000}

VALUE

- Upgrade your existing EDS with one of our high-performance systems for less than $\$ 5,000$ for MCA and Qualitative software

- Replace everything except the detector - get a new pulse processor, bias supply, MCA, quantitative software and high-performance Pentium II computer for less than $\$ 20,000$

\section{HARDIVARE PERFORMANCE}

- Total computer control - no pots to adjust

- Multiple, computer selectable shaping time constants: 3 usec for dot mapping, $12 \mu \mathrm{sec}$ for older detectors, 24 usec for modern detectors

- Super fast $2.7 \mu$ sec ADC with up to 4,000 channel resolution

- Extensive Dot Mapping Capabilities - 16 pulse outputs

- Multiple dead time methods, 9 preset modes

SOFTIVARE PERFORMANCE

- Powerful Microsoft Windows ${ }^{\mathrm{TM}}$ Environment

- Easy to use Semi-Quantitative and Quantitative versions

- Deal with eight spectra simultaneously, acquire one, analyze others

- Background modeling

- Multiple analysis approaches:

- True fundamental parameters, no-standards analysis

- Mixed mode analysis with one or a few standards

- Full standards based analysis

- User selectable X-ray databases and theoretical models

SERVICE \& SUPPORT

- Three year warranty!You can have confidence in an ANS system!

- Free or low cost software upgrades

- Support by www, e-mail, FAX or phone - talk to someone who really knows the system and EDS

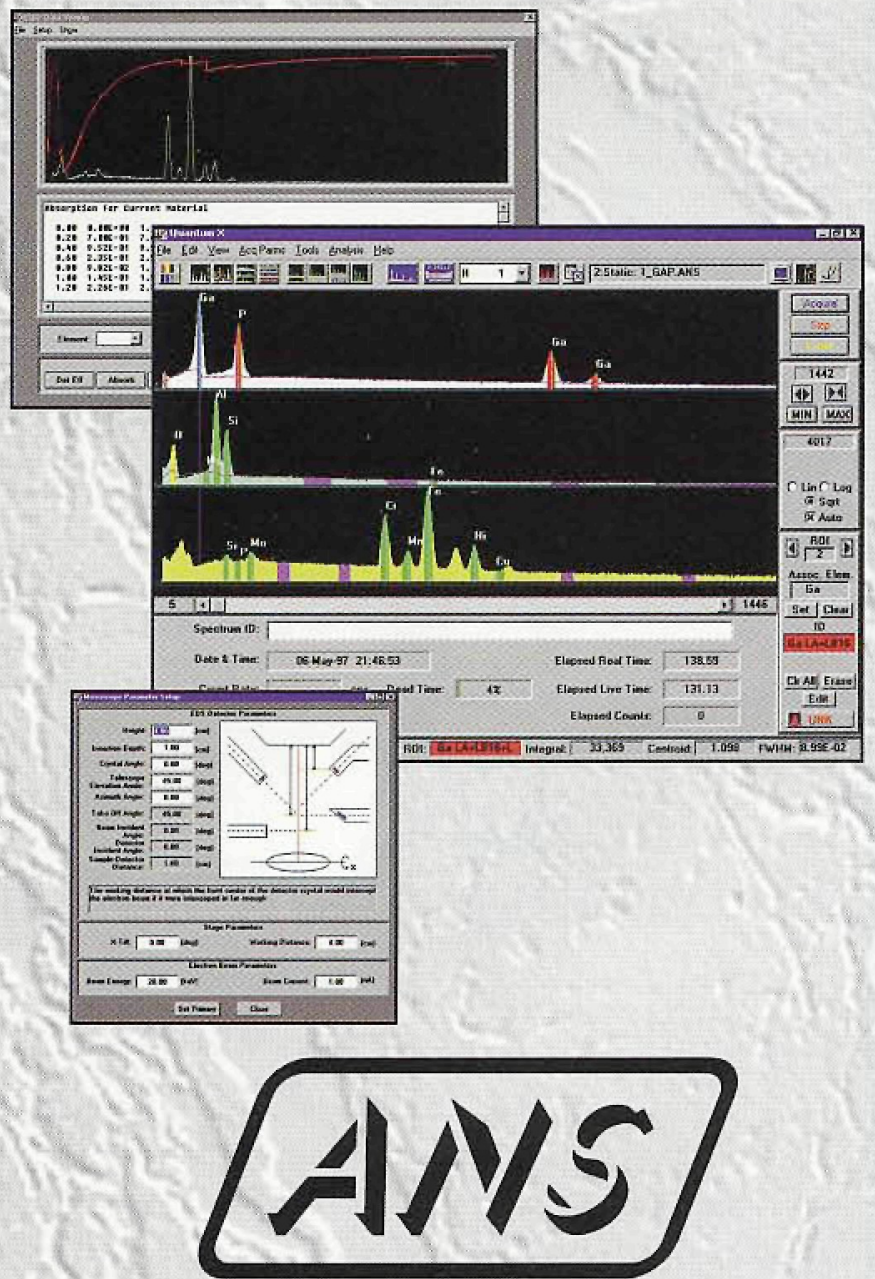

American Nuclear Systems, Inc. 1010 Commerce Park Drive, Suite G Oak Ridge, TN 37830

Tel: (423) 482-1665

FAX (423) $482-6253$

Toll Free 1-800-980-9284

E-mail: sales@qtmsys.com www.qtmsys.com 